\title{
Haemagglutination kinetics using a continuous-flow system
}

\author{
Ph ROUGER, P GANE, AND Ch SALMON \\ From the National Blood Group Reference Laboratory, 53 Boulevard Diderot, 75571 Paris Cédex 12, France
}

SUMMARY An automated technique for measuring haemagglutination kinetics is described. Equipment used in this test is very simple and already present in most blood transfusion centres. This method may be used with advantage to differentiate homozygous and heterozygous subjects in Rhesus, MNSs, and Kell systems, and to study antigen variations, especially in genetic investigations.

The antigen-antibody reaction may be studied mainly in two phases: either at the beginning of the reaction or in the steady state. Previous studies showed that the speed of the agglutination reaction was proportional to the antigen site density. In the $\mathrm{Rh}$ system, Masouredis ${ }^{1}$ and Rochna and HughesJones $^{2}$ showed that the number of $\mathrm{D}$ sites was partly a function of the genotype. A semiquantitative method was described by Lopez et al. ${ }^{3}$ An electronic particle counter, operating according to the aggregate exclusion method, can be used but the process is time-consuming and allows the study of only a few samples. ${ }^{4}$ Monnet and $\mathrm{Cabadi}^{5}$ described a rapid automatic recording method but they used an instrument derived from an aggregometer (used in the measurement of blood platelet aggregation). Our aim was to develop a rapid automatic method requiring simple equipment already present in most blood transfusion centres.

Received for publication 31 January 1980

\section{Material and methods}

The measurement of agglutination kinetics was undertaken using an AutoAnalyzer. The manifold is shown in Figure 1. It is derived partly from the manifolds used for red cell typing or antibody screening according to the method previously described by Rosenfield and Haber. ${ }^{6}$

\section{REAGENTS}

The cell samples, freshly collected in ACD or thawed, were washed in an isotonic solution and were then bromelin (or trypsin) treated as follows: one volume of bromelin solution $(2.5 \mathrm{~g} / 1000 \mathrm{ml})$ was added to one volume of packed red cells. After 10 minutes' incubation at $37^{\circ} \mathrm{C}$ the tube was filled with saline and then centrifuged, and the red blood cells were washed three times in saline. A red cell suspension $\left(4.5 \times 10^{12} / 1\right)$ was prepared.

All the antibodies (6 anti-D, 2 anti-E, 2 anti-e

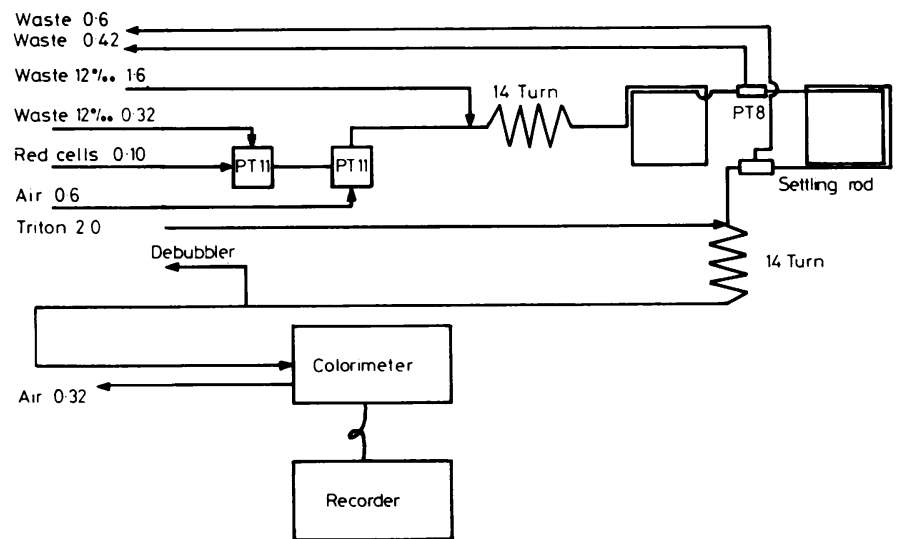

Fig. 1 Schematic diagram of manifold used for measurement of agglutination kinetics. 
2 anti-C, 2 anti-c, 2 anti-M, 2 anti-N, 3 anti-S, 3 anti-s, 3 anti-K) used were selected by preliminary tests of haemagglutination kinetics. Most of the antisera were commercial reagents used routinely. The most important problem was the choice of the appropriate dilution.

\section{PROCEDURE}

At time $0,50 \mu 1$ of the diluted antiserum were mixed with the red cell suspension $(2 \mathrm{ml})$. The mixture was immediately pumped into a reaction coil. The reaction was performed in 150 seconds. After a double decantation and lysis of the residual red cells, the haemoglobin concentration was determined in a colorimeter at continuous flow (SC ColorimeterTechnicon). The corresponding curve is shown in Figure 2. The same procedure was performed for each kinetic study. To determine the zygosity of one or more subjects, we studied in the same experiment four 'homozygous' controls, four 'heterozygous' controls, and one 'negative' control.

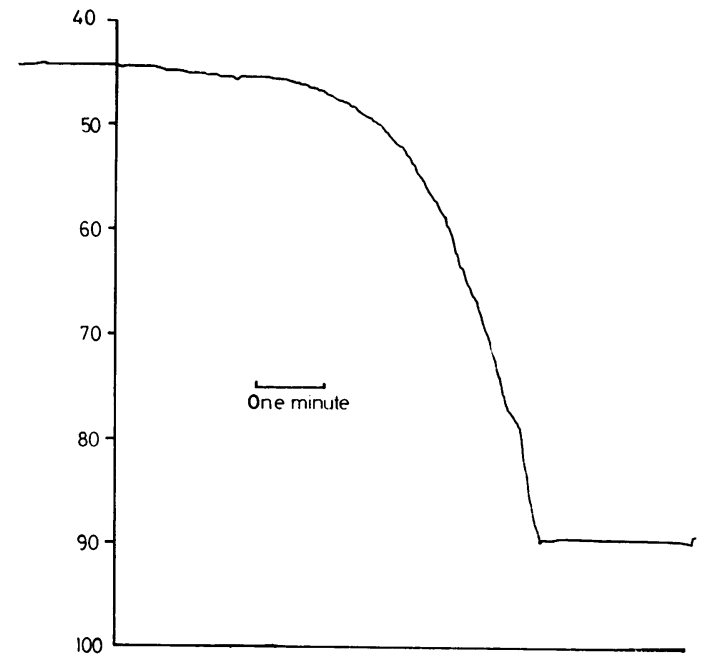

Fig. 2 Example of a curve obtained with anti-c (against DCcee red cells).

\section{Results}

All the curves were drawn on the same chart paper, and the corresponding 'homozygous zone' and 'heterozygous zone' were outlined (Figs 3 and 4). Thus, it may be clearly determined whether the curve obtained with unknown red cells corresponds to one of these two zones.

RHESUS SYSTEM

A dose effect was demonstrated by this technique

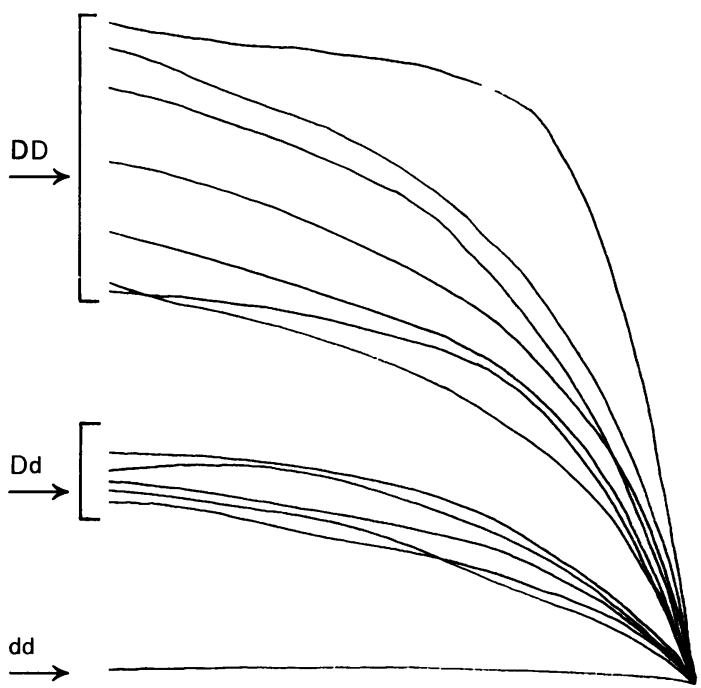

Fig. 3 Agglutination kinetics at continuous flow with anti-D.

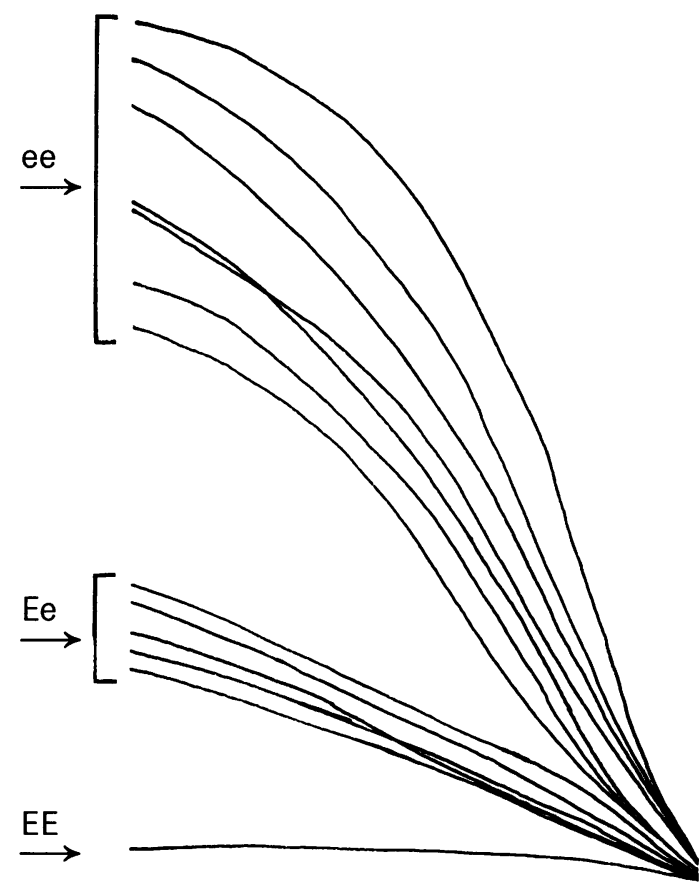

Fig. 4 Agglutination kinetics at continuous flow with anti-e.

with anti-D, anti-C, anti-c, anti-E, and anti-e. However, all the antibodies used did not give a dose effect. For 30 different samples the zygosity D/ /D or $\mathrm{D} / / \mathrm{d}$ was estimated, according to the most 
probable genotype obtained using anti-C, anti-c, anti-E, anti-e, and antibodies against compound antigens, or by family studies. No discrepancy was noted.

In a comparative study of some samples with the method described by Lopez ${ }^{3}$ no discrepancy was found. In both techniques some $R_{0} r$ samples can give a curve almost identical with $\mathbf{R}_{2} \mathbf{R}_{2}$.

\section{MNSS SYSTEM}

With this procedure, only some commercial anti-N, anti-M, anti-S, and anti-s give a dose effect.

\section{KELL SYSTEM}

The interpretation was very simple. With this method one can determine the heterozygous individuals (k/- or K/-) of a Ko family (Fig. 5).

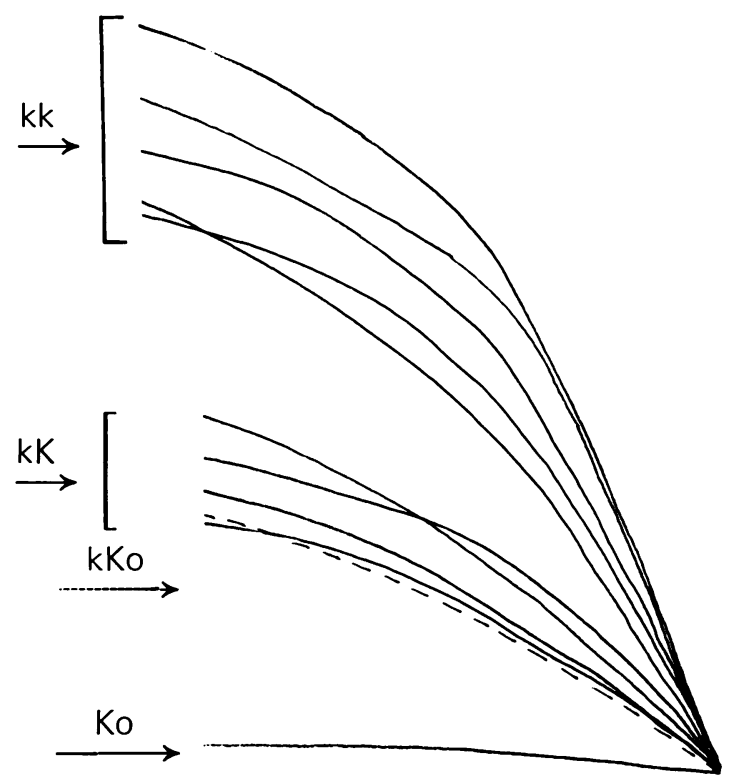

Fig. 5 Agglutination kinetics at continuous flow with anti-k.

\section{Discussion}

This method may be used to follow the first steps of an immunological agglutination reaction. The continuous recording makes it possible to detect fleeting phenomena which could not be observed using repeated punctual titrations.

It is very suitable for studying the zygosity of a red cell character: it is thus possible to determine if a $\mathrm{D}+$ subject is genetically $\mathrm{D} / \mathrm{D}$ or $\mathrm{D} / \mathrm{d}$ (or $\mathrm{D} / \mathrm{/}$ )). The method is very practical and fast in the study of a father's rhesus genotype in cases of haemolytic disease of the newborn. It is also more sensitive and remarkably more reproducible than the classical method of agglutination scoring. In our type of study, four controls of the same and genetically known phenotype must be used.

Other important applications of this technique could be the study of various parameters involved in the first step of the agglutination mechanism, mainly $\mathrm{pH}$, ionic strength, temperature, macromolecules, enzymes, and antibody affinity.

\section{References}

${ }^{1}$ Masouredis SP. Relationship between $\mathrm{Rh}_{0}$ (D) genotype and quantity of ${ }^{131} \mathrm{I}$ anti-R $\mathrm{h}_{0}$ (D) bound to red cells. J Clin Invest $1960 ; 39: 1450-62$.

${ }^{2}$ Rochna E, Hughes-Jones NC. The use of purified ${ }^{125} I$ labelled anti- $\gamma$ globulin in the determination of the number of $\mathrm{D}$ antigen sites on red cells of different phenotypes. Vox Sang 1965;10:675-86.

${ }^{3}$ Lopez M, Habibi B, Lemeud J, Salmon Ch. Agglutination kinetics: a method for quantitative red cell antigen assays. Vox Sang 1975;28:57-61.

${ }^{4}$ Solomon JM, Gibbs MB, Bowlder AD. Methods in quantitative hemagglutination. Vox Sang 1965;10:133-48.

${ }^{5}$ Monnet A, Cabadi Y. Automatized hemagglutination kinetics. Vox Sang 1978;34:227-30.

- Rosenfield RE, Haber GR. Detection and measurement of homologous human hemagglutinins. In Automation in Analytical Chemistry. New-York: Technicon Symposium, 1965.

Requests for reprints to: Dr Ph Rouger, National Blood Group Reference Laboratory, 53 Boulevard Diderot, 75571 Paris Cédex 12, France. 